\title{
Quantifying nanoparticle cellular uptake: which method is best?
}

\author{
"...the method of choice for the quantification of \\ nanoparticle uptake mainly depends on the research \\ question, the available analytical devices as well as on the \\ type of nanoparticles of interest."
}

First draft submitted: 28 February 2017; Accepted for publication: 9 March 2017; Published online: 27 April 2017

Keywords: chemical analysis $\bullet$ detection $\bullet$ electron microscopy $\bullet$ fluorescence-based techniques $\bullet$ intracellular nanoparticles $\bullet$ quantification

As the range of engineered nanoparticles (NPs) designed as specific carriers increases, for example for cell targeting and drug delivery, the question on how many NPs are interacting or are taken up by cells is becoming increasingly important for any potential biomedical application. On one hand, the delivered dose of such NPs to the targeted cells is a key parameter in the assessment of their efficiency to perform the desired action (e.g., deliver the therapeutic substance or induce a specific effect), on the other hand, the assessment of intracellular NPs is crucial also from the safety aspect as NPs might come unintentionally in contact by untargeted cells. Particularly from the regulative perspective, it is important that reproducible and reliable analytical methods for the intracellular quantification of NPs are available at an early stage in the development in order to correlate the cell burden of NPs with their possible effects at a cellular level.

\section{Which method is the best?}

The authors approached this matter by questioning senior scientists in the field of nanoscience about their personal views on the prime method for quantifying intracellular NPs. Although - at first glance - this is a seemingly straightforward enquiry, their responses were rather ambiguous yet consistent: in a nutshell, they all concluded the method of choice for the quantification of NP uptake mainly depends on the research ques- tion, the available analytical devices as well as on the type of NPs of interest. As of that, it is not possible to recommend one specific technique that could be used for quantification of all the different NPs types which exist nowadays. As well known from the convincing evidence from the literature, physicochemical properties of NPs such as their size, shape, core material and surface functionalization have a strong impact on NP cellular interaction including uptake, intracellular fate and induction of cell response but also require very different analytical methods. Various cutting-edge techniques have emerged during the last years which can quantify the NPs based on their distinctive characteristics, for example - chemical composition, optical properties, magnetic properties or electron density. Comprehensive reviews and in-depth discussions are readily available [1-4] and are not reiterated herein. For the purpose of this commentary, we have selected some of the most widely used analytical techniques in the nanoscientific biomedical community based on a review of the existing nanotoxicology studies published within the scope of the last 5 years. Noteworthy, we do not differentiate here between intracellular and cell associated NPs which can of course also influence the choice of a method, however, it is an important point which has to be considered for the interpretation of the result. And it is important to add that, depending on the community and the research field, quanti-
Barbara Drasler ${ }^{\ddagger 1}$, Dimitri Vanhecke ${ }^{\ddagger 1}$, Laura RodriguezLorenzo', Alke Petri-Fink ${ }^{1,2}$ \&

Barbara Rothen-Rutishauser *,1 ${ }^{1}$ Adolphe Merkle Institute, University of Fribourg, Chemin des Verdiers 4, 1700 Fribourg, Switzerland ${ }^{2}$ Department of Chemistry, University of Fribourg, Chemin du Museé 9, 1700 Fribourg, Switzerland *Author for correspondence: Tel.: +41263009502 barbara.rothen@unifr.ch ${ }^{\ddagger}$ Authors contributed equally 
fication per se can have quite different meanings. In general, quantification is described as the act of giving a numerical value to a measurement, including the calibration of a system (method and instrument) and scale formation [5]. From an analytical chemistry point of view, the term quantification can be explained as the process of determining a quantity of (an) analyte(s), for example - chemical species, present in a sample. In cell biology, quantification is often described with the attributes of the measurement itself (e.g., accuracy and precision) whereas in medical and pharmaceutical community the term quantification is usually correlated with the procurement process of a medicine/ drug.

\section{General considerations about quantification techniques}

First of all, the unambiguous detection of NPs in a cell is a prerequisite for the subsequent quantification. Because of the small particle size and consequently very low mass and along with the fact that in some cases only a small number of NPs might be associated with cells, detection within cells is extremely challenging due to the sensitivity or resolution limits for some of the techniques. Noteworthy, detection of NPs in the biologically complex environment is reasonably required in order to distinguish the particles from either other cellular parts of the similar sizes, for example ribosomes or glycogen, or from other xenobiotic material coincidentally present in the cells. Moreover, one should also consider the different and constantly changing biological environments with respect to, for example - presence of proteins and electrolytes, different $\mathrm{pH}$ values etc, that NPs can encounter when finally localized in various subcellular compartments such as cytosol, endosome or endolysosomes; all this might importantly influence the measurements and render quantification very challenging.

66

"...quantification is described as the act of

giving a numerical value to a measurement, including the calibration of a system (method and instrument) and scale formation."

Only once NPs are detected inside the cells, quantification can be performed [6], which yields numerical values from the measurement. The obtained value can then be converted into different quantities, for example - NP mass, NP number or NP surface per reference volume which can be a cell population, number or volume unit $\left(\mu \mathrm{m}^{3}\right)$.

In this context, the result from a quantitative technique depends on the quality of parameters among others, the accuracy, precision, sensitivity and resolution. To start with, accuracy of measurement of a device is the range of the statistical bias and how close it gets to the actual value, while the precision indicates the reproducibility and repeatability of the measurement. Precision also reflects the heterogeneity of an object and can be reliably estimated by repeated experiments. Both the sensitivity and the resolution, defining the smallest quantity and the smallest object that can be detected, respectively, are highly method dependent, hence these limitations are difficult to be overcome and improved by the researcher. These method parameters are manifested in the overall quality and performance of the respective quantitative analytical tools; however, there are also other criteria which are important for the quality of these techniques including the validation of the measurement with standard samples. All these criteria are in concordance with the US FDA requirements for general development and establishment of sound bioanalytical methods [7] including a detailed description of the analytical technology, target analyte (e.g., the NP) and the sample (e.g., the cell type).

Another key requirement for the reliable quantification of NPs per cell is to reflect the biological variability. This does not only refer to the obvious differences among the various cell types existing (e.g., plant vs. mammalian cells, prokaryotic vs. eukaryotic cells), but also on the possible differences in the uptake of NPs within one cell population because of different cell cycle phases. High fluctuations of intracellular load of NPs have been reported populations in in vitro cell culture studies using human alveolar epithelial [8] and human urinary bladder cell lines [9]. This is the reason why the prerequisite for a representative cell sample size is also of utmost importance in order to produce accurate and precise measurement values. Finally, an efficient and reliable quantification entails unbiased sampling, that is - randomly chosen measuring spots in the sample, meaning that any object in the population must have equal chances to be measured.

Having noted all this, it is still questionable on how all these principles can practically be manifested in a real research environment? And which decision-making process on the selection of quantitative method should be followed - given the fact that quantification depends on both the NPs' intrinsic characteristics and the desired quantification data? Unfortunately, there is still no simple answer available, yet it can be illustrated by giving some examples of available techniques for a chosen set of NPs relevant for the biomedical field.

\section{NP quantification techniques: some illustrative examples}

When NP cell interactions are assessed, the properties of both, the particles as well as the cellular structures, should be preserved. As already said, the quantifica- 
tion method of choice for the intracellular detection and quantification of NPs also highly depends on the characteristics of the particles themselves - chemical composition, surface properties, spectroscopic properties (e.g., fluorescence and scattering), size and shape.

The analytical techniques are diverse and mainly include spectroscopic and imaging methods. Inductively coupled plasma (ICP)-based spectroscopic techniques including -optical emission spectrometry (ICP-OES) [10], as well as mass spectrometry (ICPMS) [11] can provide quantitative elemental composition and is applicable for a wide range of NPs such as metals, (metal) oxides or quantum dots [12]. The major advantage of these techniques is the very sensitive detection range; namely, the sensitivity of ICP-MS exceeds that of ICP-OES (ppt to ppm range, respectively) and ICP-MS is also capable of providing isotope information. Moreover, the costs of both installing the facilities and development of specific methodology are lower for OES with respect to MS, yet the individual sample running times are shorter with MS; nonetheless, measurements itself are rather fast with both the approaches. However, one has to keep in mind that the destructive nature of the techniques for particles as well as the cellular structures which does not allow differentiation between NPs and metal ions, distinction between internalized NPs, extracellularly associated and/or just located between cells or obtain spatial information [13]. These methods do not allow for the determination of mass in a single cell but it usually refers to a cell population. In cell culture assays, it is, however, possible to determine the cell number per sample size and thus extrapolate the NP quantities per cell number. However, ICP-MS has been advanced as single particle ICP-MS, which enables the distinction between the dissolved and particulate form of NPs, yet is it has not been revealed to what extend the complex biological matrix affects the signal produced from an NP and hence influence the measurement; the technique requires further development [14]. Nevertheless, such an approach for quantification of mass using elemental analysis techniques is a reliable tool to assess the material mass within a defined sample volume.

Fluorescence-based quantitative techniques provide again highly sensitive methods as only a few or even individual fluorochromes can be detected. These techniques require a fluorescence signal from the NPs which can derive from an intrinsic property of NPs exhibiting the capacity to emit the light, for example semiconductor nanocrystals such as quantum dots [15] and titanium dioxide NPs [16] or by the use of fluorescence tags [17]. These types of NPs can be detected and quantified by fluorescence spectroscopy, flow cytometry, or fluorescence imaging techniques. However, the resolution limits of all these methods do not allow to resolve single NPs. Herein, the NP load per cell is typically expressed as an indirect marker such as a number of events or intensity of the signal associated with NPs and this also strongly depends on the resolution of the detector. In flow cytometry, single cells pass in a steady stream in front of a laser detection unit that collects the fluorescent signals from a single cell on appropriate detectors. The integrated fluorescent signal from individual cells is measured by side scattering and interpreted as either NPs containing cell or NPs free cell. The signal integration increases the sensitivity compared with fluorescence imaging methods, where the fluorescence signal is spread out over millions of camera pixels, but is also the main drawback: the relative location of the NPs in the cell is lost. Forward scattered light is useful to determine the volume of the cell, thus the reference volume is defined in this specific technique. It is a fast method to obtain the relative amount of cells loaded and/or associated with fluorescently labeled NPs (or possessing fluorescent properties) $[16,17]$. In addition, the cells have to be labeled in order to define the reference volume. In fluorescence microscopy, the quantification of events is usually created based on digital images. The events can be counted manually or by an automated analysis in silico following an image segmentation process. Yet, a clear distinction between the NPs and the biological material needs to be assured, meaning that the NP-derived signal must exceed the autofluorescence-induced noise. Typically, the reference space can be assessed using a separate recording channel with a cell or a cellular compartment specific fluorescent labeling.

\section{"The analytical techniques are diverse and mainly include spectroscopic and imaging methods.}

Fluorescent quantification methods do not account for possible bleaching or quenching of the fluorescent signal at locally high concentrations of the fluorochrome and are easily prone to artifacts leading to inaccurate quantification [18]. The common feature of all the fluorescence-based methods is the fact that the fluorescence signal is not absolute because the signal intensity depends on the excitation source, the number of fluorophores per NP, quantum yield of fluorophores or the NP itself and the sensitivity of the detector. Therefore, the signals are compared with a control sample and are given as relative values, that is, semiquantitative outcomes. Thus, fluorescence methods neither indicate mass nor NP number, but they allow the comparison between different experimental conditions.

Adequate resolution of an analytical method is the prerequisite for the quantification of absolute particle 
number or surface per cell which can be provided by electron microscopy (EM) techniques (e.g., scanning EM [SEM], focused ion beam [FIB]-SEM, transmission EM [TEM]). An EM micrograph provides the signal of electron dense NPs and the biological context within one channel which requires interpretation prior to quantification. Therefore, computer-assisted counting is often not an option. With increased human intervention in the interpretation, observer expectancy effects may affect the accuracy. The EM techniques provide information in $2 \mathrm{D}$, that is - surface by SEM and projection by TEM or in 3D obtained by for example FIB-SEM, TEM tomography or serial TEM sections. Accurate quantification requires for all NPs to have the same chance of being sampled, but the sampling chance is proportional to NPs size: the larger the particles, the greater the chance for being counted in $2 \mathrm{D}$ projections. A uniform chance - and with it the accuracy - can only be assured with a $3 \mathrm{D}$ probe. This also derives intuitively from the observation that if the aim is to quantify numerical density a volume must be sampled.

The dissector [19] is a stereological approach which registers the presence of NPs in a reference section and compares this with the presence of the NPs in deeper sections, allowing accurate numerical density estimation, independent of NP size and morphology. When multiplied by the reference volume, NPs cardinality is obtained [20,21]. Besides the dissector, additional stereological approaches exist that deliver unbiased estimates of other units of interest. For the volume, the Cavalieri principle can be useful for reference space (cell or cell compartment) estimation. For NPs surface estimation, a number of approaches are available such as isotropic fakir probes or the surfactor probe [22]. Stereological EM studies yield accurate NP number and surface information in a relative efficient way, if applied properly. However, the design of the experiments, including the sampling schemes and sample preparation, can be a time consuming task compared with other approaches discussed above.

Stereological methods are not limited to EM modalities but can be applied to other imaging 3D datasets. They require parallel sections of constant thickness: either physical (e.g., FIB-SEM, serial light microscopy [LM] or TEM sections) or optical (TEM tomography, confocal laser scanning microscopy). Naturally, the resolution of the imaging modality will influence the precision of the quantification. Additionally, an accurate estimation is assured if the requirements of the estimator are fulfilled. Surface estimation methods such as the surfactor require uniform isotropic random sections, which are random in all three planes whereas the isotropic fakir and Cavalieri method requires the less demanding preferential sectioning. Finally, systematic random sampling schemes provide ways to select the NPs without bias (or the known bias) regarding the size or orientation.

The sampling requirements and counting schemes may be demanding for certain EM techniques. Comparing multiple serial TEM sections simultaneously is challenging, but may be converted to an offline task by TEM tomography. Keeping systematic random sampling schemes in a FIB-SEM may not be practical due to the long acquisition time involved (several hours per data point). If the time component is not an obstacle, the EM combined with stereological methods provide accurate estimates of difficult to obtain measures, such as NP number and NP surface, which are often crucial to explain the NP interaction with the biological context.

\section{Final comments}

The quantification of NPs internalized by cells in dependence of the NP properties is of vital importance in the emerging field of 'bionanomedicine'. Up to date there is no standardized and validated method available for this specific question because of the very different requirements of the analytical procedure depending on the particle type, the biological environment as well as the different specifications and limitations for each of the techniques. Given all the above stated thoughts, it is not possible to provide a clear answer on the best method to be employed in NP uptake quantification and we also did not cover all available methods but tried to focus on the most applied ones. For a reliable determination of the intracellular NP burden it is recommended to combine different methods yielding complementary outcomes, such as efficient mass or number estimations supported by a visual evidence of the NPs localization inside the cells. Regardless of the approach employed one also has to consider that every quantitative measurement contains some amount of the error and the limitations of the systems have to be taken into account for the interpretation of the data.

Financial \& competing interests disclosure

This work was supported by the Adolphe Merkle Foundation, the Swiss National Science Foundation through the National Centre of Competence in Research 'Bio-Inspired Materials' and the grant no. 310030_159847/1. The authors have no other relevant affiliations or financial involvement with any organization or entity with a financial interest in or financial conflict with the subject matter or materials discussed in the manuscript apart from those disclosed.

No writing assistance was utilized in the production of this manuscript. 
Open access

This work is licensed under the Attribution-NonCommercial-
NoDerivatives 4.0 Unported License. To view a copy of this license, visit http://creativecommons.org/licenses/by-nc-nd/4.0/

\section{References}

1 Elsaesser A, Taylor A, De Yanes GS et al. Quantification of nanoparticle uptake by cells using microscopical and analytical techniques. Nanomedicine 5(9), 1447-1457 (2010).

2 Vanhecke D, Rodriguez-Lorenzo L, Clift MJD, Blank F, Petri-Fink A, Rothen-Rutishauser B. Quantification of nanoparticles at the single-cell level: an overview about stateof-the-art techniques and their limitations. Nanomedicine 9(12), 1885-1900 (2014).

3 Collins AR, Annangi B, Rubio L et al. High throughput toxicity screening and intracellular detection of nanomaterials. Wiley Interdiscip. Rev. Nanomed. Nanobiotechnol. 9(1), doi:10.1002/wnan.1413 (2017) (Epub ahead of print).

4 Rashkow JT, Patel SC, Tappero R, Sitharaman B. Quantification of single-cell nanoparticle concentrations and the distribution of these concentrations in cell population. J. R. Soc. Interface 11(94), doi:10.1098/rsif.2013.1152 (2014) (Epub ahead of print.)

5 Williams JH. The tyrany of numbers. In: Quantifying Measurement. Morgan \& Claypool Publishers, CA, USA, 4433-4439 (2016).

6 Wilks SS. Some aspects of quantification in science. Isis 52(168), 135-142 (1961).

7 Tiwari G, Tiwari R. Bioanalytical method validation: an updated review. Pharm. Meth. 1(1), 25-38 (2010).

8 Kim JA, Aberg C, Salvati A, Dawson KA. Role of cell cycle on the cellular uptake and dilution of nanoparticles in a cell population. Nat. Nano. 7(1), 62-68 (2012).

9 Jeynes JCG, Jeynes C, Merchant MJ, Kirkby KJ. Measuring and modelling cell-to-cell variation in uptake of gold nanoparticles. Analyst 138(23), 7070-7074 (2013).

10 Montaser A, Golightly DW. Inductively Induced Coupled Plasmas in Analytic Atomic Spectra (2nd Edition). WileyBlackwell, NJ, USA, 1040 (1992).

11 Taylor H. Inductively Coupled Plasma-Mass Spectrometry. Academic Press, MA, USA, 294 (2000).
12 Krystek P, Ulrich A, Garcia CC, Manohar S, Ritsema R. Application of plasma spectrometry for the analysis of engineered nanoparticles in suspensions and products. J. Anal. Atomic Spectrometry 26(9), 1701-1721 (2011).

13 Gottstein C, Wu GH, Wong BJ, Zasadzinski JA. Precise quantification of nanoparticle internalization. Acs. Nano. 7(6), 4933-4945 (2013).

14 Montano MD, Olesik JW, Barber AG, Challis K, Ranville JF. Single particle ICP-MS: advances toward routine analysis of nanomaterials. Anal. Bioanalyt. Chem. 408(19), 5053-5074 (2016).

15 Chen M, He XX, Wang KM, He DG, Yang XH, Shi H. Inorganic fluorescent nanoprobes for cellular and subcellular imaging. Trac.-Trend. Anal. Chem. 58 120-129 (2014).

16 Zucker RM, Massaro EJ, Sanders KM, Degn LL, Boyes WK Detection of $\mathrm{TiO} 2$ nanoparticles in cells by flow cytometry. Cytometry A 77(7), 677-685 (2010).

17 Shapero K, Fenaroli F, Lynch I, Cottell DC, Salvati A, Dawson KA. Time and space resolved uptake study of silica nanoparticles by human cells. Mol. BioSystems 7(2), 371-378 (2011).

18 Waters JC. Accuracy and precision in quantitative fluorescence microscopy. J. Cell Biol. 185(7), 1135-1148 (2009).

19 Sterio DC. The unbiased estimation of number and sizes of arbitrary particles using the disector. J. Microsc. 134(Pt 2), 127-136 (1984).

20 Vanhecke D, Studer D, Ochs M. Stereology meets electron tomography: towards quantitative $3 \mathrm{D}$ electron microscopy. J. Struct. Biol. 159(3), 443-450 (2007).

21 Brandenberger C, Ochs M, Muhlfeld C. Assessing particle and fiber toxicology in the respiratory system: the stereology toolbox. Part. Fibre Toxicol. 12, 1-15 (2015).

22 Gundersen HJG, Jensen EB. The efficiency of systematicsampling in stereology and its prediction. J. Microsc. 147, 229-263 (1987). 